\title{
RDDS CELL DESIGN AND OPTIMIZATION FOR THE LINEAR COLLIDER LINACS *
}

\author{
Z. Li, J. Irwin, K. Ko, R. J. Loewen, E. W. Lundahl, B. McCandless, \\ R. H. Miller, R. D. Ruth, Y. Sun, K. W. Vaillancourt, J. W. Wang, \\ Stanford Linear Accelerator Center, Stanford University, Stanford, CA 94039
}

\begin{abstract}
Each of the JLC/NLC main linacs will consist of $\sim 1$ million complex 3D accelerating cells that make up the 1.8meter Rounded Damped Detuned Structures (RDDS) along its eight kilometer length. The RDDS is designed to provide maximum accelerating gradient to the beam while being able to suppress the long-range transverse wakefields to a satisfactory level. Using the 2D finite element code, Omega2, a $15 \%$ improvement in shunt impedance is found by changing the basic cell shape from a straight cylinder to a round outer wall contour that connects to slightly bulging circular disk noses. The HOM damping manifold is then designed around this optimal cell shape to improve the cell-to-manifold coupling for the dipole mode and the vacuum conductance under the frequency and minimal Qreduction constraints for the fundamental mode. We use both MAFIA and the 3D finite element Omega3 code for this step to obtain a manifold geometry that consists of a round waveguide with additional narrow coupling slots that cut into the cell disks. As a time and cost saving measure for the JLC/NLC, the RDDS cell dimensions are being determined through computer modeling to within fabrication precision so that no tuning may be needed once the structures are assembled. At the X-band operating frequency, this corresponds to an error of a few microns in the cell radius. Such a level of resolution requires highly accurate field solvers and vast amount of computer resources. We will present calculations with the parallel code Omega3P that utilizes massively parallel computers such as the Cray T3E at NERSC. The numerical results will be compared with coldtest measurements performed on RDDS prototypes that are diamond-turned with dimensions based on Omega3P simulations.
\end{abstract}

\section{INTRODUCTION}

The Next Linear Collider (JLC/NLC) $[1,2]$ is a $e^{+}, e^{-}$machine to provide a luminosity of $10^{34} \mathrm{~cm}^{-2} \mathrm{sec}^{-1}$ or higher at a center of mass of about $1 \mathrm{TeV}$. The main linacs of the NLC use normal-conducting traveling-wave copper structures operating at X-band $(11.424 \mathrm{GHz})$ frequency. The accelerator structure will be 1.8 meter in length and will be powered by $75 \mathrm{MW}$ klystrons with pulse compression using Delay-Line Distribution Systems (DLDS)[3]. About 10,000 accelerator structures operating at a gradient of about $70 \mathrm{MV} / \mathrm{m}$ are needed to obtain the $1 \mathrm{TeV}$ center

\footnotetext{
* Work supported by the Department of Energy, contract DE-AC03$76 \mathrm{SF} 00515$.
}

of mass energy. The linacs need to accelerate long bunch trains of $266 \mathrm{~ns}$ to obtain the required luminosity. The efficiency of the accelerator structure is a major factor that drives the cost of the the rf systems and thus the linac. The issues related to the long bunch train acceleration are the short- and long-range dipole wakefields associated emittance dilution and multi-beam breakup. Thus, the design requirements for the collider linac structures are to maximize the rf efficiency and to be able to suppress the dipole wakefields. There have been continuous efforts for the JLC/NLC R\&D to build the high gradient X-band structures. A number of X-band structures based on a rightstraight cylinder cell profile (DLWG as shown in Fig. 1) have been built and tested [4]. With modern numerically controlled machining, linac cells which are spheroid of revolution are negligible more expensive than right straight cylinders. On the other hand, significant cost saving can be achieved by optimizing the structure rf efficiency. With this motivation, the cell geometry were optimized to improve the shunt impedance. Round Damped Detuned Structure (RDDS) was designed based on the optimized cell profile to minimize the long-range dipole wakefields by using detuning and damping.

The structure was first optimized on a single cell level using finite-element code Omega2 [5]. Then the dimensions of the 206 cell of the $1.8-\mathrm{m}$ structure were tailored to detune the important dipole modes. And thirdly, the damping manifold was designed using MAFIA [6] and Omega3 [7] around the detuned structure to improve the vacuum conductance and to suppress the long-range dipole wakefields. Finally, the cell dimensions of the 3D RDDS was calculated using parallel code Omega3P [7].

\section{CELL PROFILE OPTIMIZATION}

The traveling-wave structure was designed with a phase advance of $120^{\circ}$ per cell. A number of modifications on the cell profile were made to improve the shunt impedance. Firstly, the rectangular cylinder cell was replaced by a revolution of a round contour, which improves the $\mathrm{Q}$ for the fundamental mode by about $10 \%$. Then, the tip of the iris was reshaped to include a slight bulging to improve the $R / Q$. The cell profile of such a design is shown in Fig. 1. The top arc of the cell is a full half circle. The arc connecting the iris and the straight section in the disk has the same radius as the top arc. The peak surface E field appears on the surface of the round iris and is controlled by the bulging radius. The disk thickness $t$ can be thinner than in a standard DLWG cell to improve the $R / Q$ without en- 
hancing the peak surface field. With the optimized profile, the average shunt impedance of the structure is about $15 \%$ higher than that of the DLWG structure. A comparison of the rf parameters of three representative cells in the detuned structure is shown in Table 2.
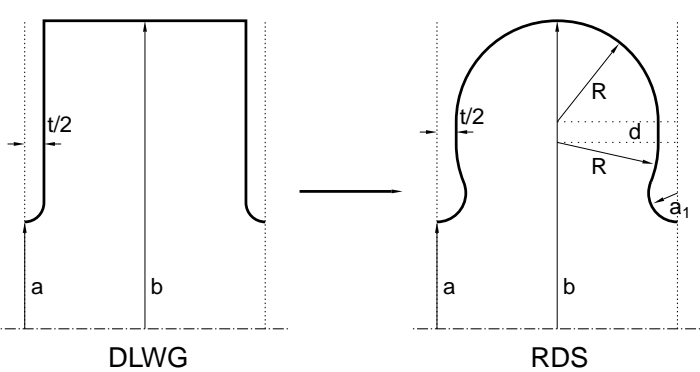

Figure 1: Disk loaded waveguide cell shapes.

Table 1: Comparison of DLWG and RDS RF parameters

\begin{tabular}{|c|c|c|c|c|c|}
\hline Cell & Type & $\mathrm{R}\left(\frac{M \Omega}{m}\right)$ & $\mathrm{Q}$ & $\mathrm{R} / \mathrm{Q}$ & $\frac{E_{s}}{E_{a c c}}$ \\
\hline \hline \multirow{2}{*}{001} & RDS & 76.9 & 8300 & 9260 & 3.01 \\
\cline { 2 - 6 } & DLWG & 66.7 & 7344 & 9219 & 3.11 \\
\hline \hline \multirow{2}{*}{103} & RDS & 90.7 & 7847 & 11556 & 2.41 \\
\cline { 2 - 6 } & DLWG & 79.7 & 7001 & 11386 & 2.43 \\
\hline \hline \multirow{2}{*}{206} & RDS & 103.5 & 7463 & 13866 & 2.19 \\
\cline { 2 - 6 } & DLWG & 88.8 & 6621 & 13413 & 2.09 \\
\hline
\end{tabular}

\section{CELL DIMENSIONS OF DETUNED STRUCTURE}

To suppress the long-range dipole wakefield, the dimensions of the 206 cells in the structure are tailored to detune the dipole modes while maintaining the fundamental mode frequency at $11.424 \mathrm{GHz}$. The modes that have large contributions to the transverse wakefields are in the first, the third and the sixth dipole bands. The detuning of the dipole modes were realized by detuning the iris opening $a$ for the first band, the disk thickness $t$ for the third band, and the iris bulging radius $a_{1}$ for the six band. While varying $a, t$, and $a_{1}$ along the structure to detune the dipole modes, the cell radius $b$ is adjusted accordingly to obtain $11.424 \mathrm{GHz}$ for the fundamental mode. The average iris radius is determined by the short-range wakefield related structurestructure tolerance requirements as $a_{\text {ave }}=0.18 \lambda$, where $\lambda$ is the rf wave length. The minimum disk thickness $t$ is limited to $0.75 \mathrm{~mm}$ due to mechanical considerations. Among the three dipole bands, the modes in the first dipole band dominate the transverse wakefields. The transverse kick factors $k d n / d f$ of this band is detuned in a Gaussian manner as shown in Fig. 2, so that the dipole wakefields drop, following a Gaussian profile, by a factor of 100 within a distance of 0.42 meters due to de-cohere cancellations. The total detuning of the spectrum is $11.25 \%$, in $4.75 \sigma \mathrm{s}$, of the center frequency $15.2 \mathrm{GHz}$. Each of the dipole frequencies in the spectrum corresponds to one cell of the detuned structure. The third and sixth dipole bands are detuned with a similar detuned range. The cell and rf parameter distributions along the round detuned structure (RDS) are shown in Fig. 3.

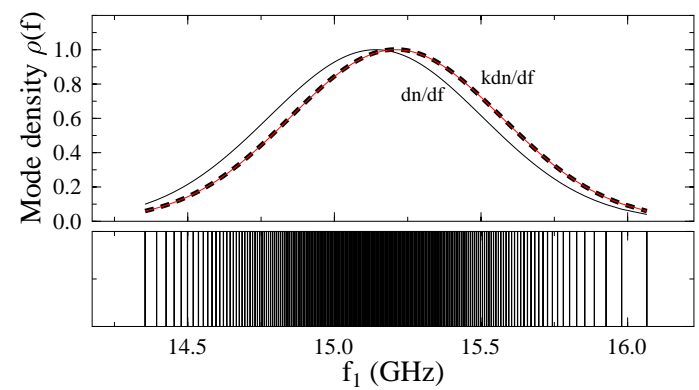

Figure 2: RDS structure dipole spectrum. $k d n / d f$ is the density of the transverse kick factor and $d n / d f$ is the modal density.
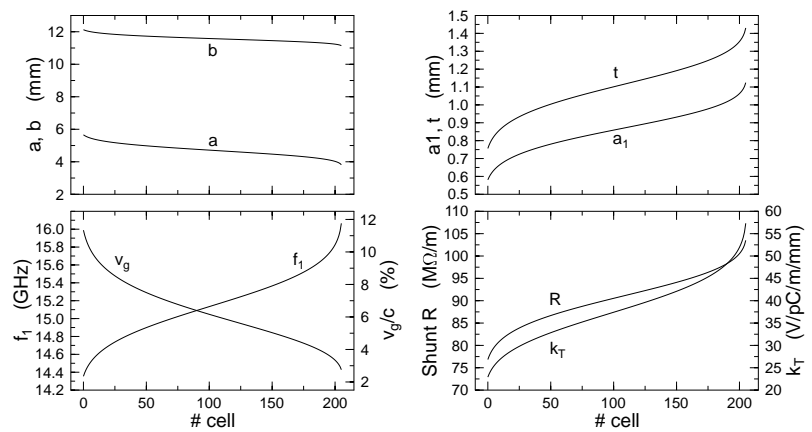

Figure 3: RDS structure cell dimensions and rf parameters.

\section{ROUND DAMPED DETUNED STRUCTURE (RDDS)}

The damping manifolds were designed on top of the optimized round detuned structure. The goals for the RDDS design were to improve the longitudinal vacuum conductance, to minimize the $\mathrm{Q}$ reduction for the fundamental mode, and to obtain adequate coupling for the dipole modes. As compared with the DDS1 [8], a number of new features were incorporated into the RDDS as shown in Fig. 4: firstly the rectangular manifold is replaced by a round manifold to maximize vacuum conductance, secondly narrow slots cutting into the disk are added to enhance the dipole mode coupling, and finally wide slots were added to raise the cutoff of the manifold mode (eliminating the ridged waveguide effect due to only a narrow slot), which allows a large round manifold and further improves the vacuum. The manifold dimensions were designed to cutoff frequencies bellow $12 \mathrm{GHz}$ to avoid coupling to the fundamental mode. The narrow slot width is chosen to be $1.5 \mathrm{~mm}$ to minimize the effect on the fundamental mode. The longitudinal dimension of the wide slot is $6 \mathrm{~mm}$. The width of the wide slot is optimized to minimize the $Q_{0}$ 
reduction of the fundamental mode due to the slot openings. Fig. 5 shows the rf parameters as functions of the wide slot width. A $3 \mathrm{~mm}$ wide slot was adopted for the current RDDS. The $Q_{0}$ reduction with such a design is less than $1 \%$. The vacuum conductance of the RDDS structure is over 4 times better than a pure detuned structure. An appreciable dipole coupling was obtained with a $3 \mathrm{~mm}$ narrow slot depth, however the final narrow slot depth will be determined by the damping requirements for the long-range wakefields [9].

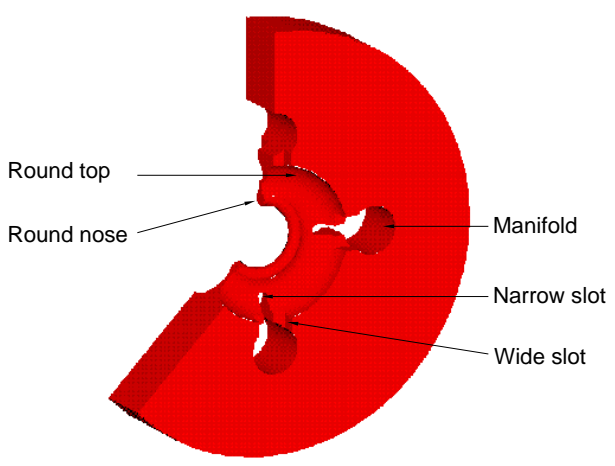

Figure 4: Damped RDDS structure cell.
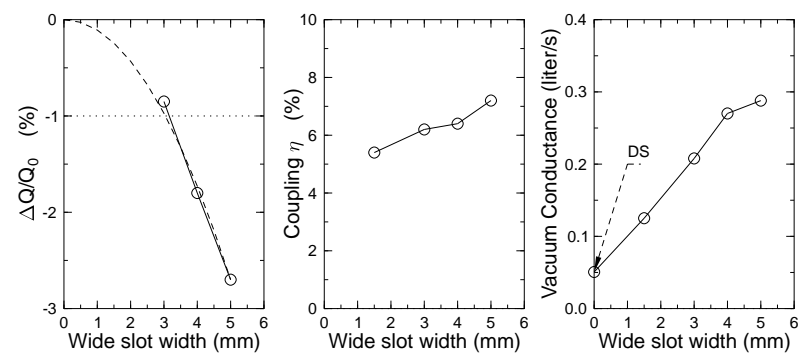

Figure 5: Optimization of wide-slot width for the RDDS. The length of the wide-slot is $6 \mathrm{~mm}$ and the depth of the narrow-slot is $3 \mathrm{~mm}$ in the simulations. The vacuum is estimated for one meter uniform RDDS with cell-206 parameters.

\section{RDDS DIMENSION CALCULATION}

A time and cost saving approach to determine the 3D RDDS dimensions is through computer modeling. The frequency tolerance on the fundamental mode of the $\mathrm{X}$ band structure is about $1 \mathrm{MHz}$ in order to maintain a better than $98 \%$ acceleration efficiency in the structure. At the $11.424 \mathrm{GHz}$, this tolerance corresponds to a few microns in the cell dimensions. To model the RDDS with such an accuracy, vast computer resources and accurate field solvers are required. This paper is the first attempt to use the newly devolved finite-element parallel code Omega3P to design the RDDS to an accuracy comparable to the hardware machining tolerance. The finite-element mesh to model the RDDS cell is as shown in Fig. 6, which has about one million degrees of freedom. The typical run time on such a grid using 48 processors on the NERSC T3E parallel supercomputer is less than half an hour. Fig. 7 shows the Omega3P results for the frequency shift due to the damping manifolds. The cell dimensions were adjusted based on these frequency shifts. The cell dimensions obtained by using Omega3P is within a couple of microns accuracy as compared with the coldtest results. The accuracy and turnaround time for modeling such a complicated geometry are unprecedented. Further improvement in accuracy can be achieved by reducing the element size in the model.

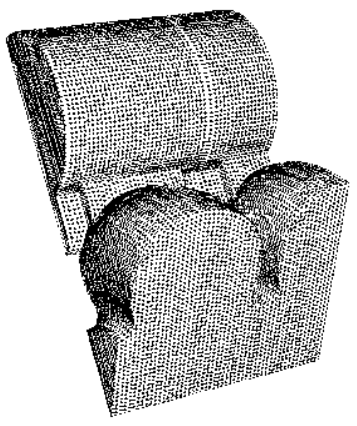

Figure 6: Finite element mesh for modeling RDDS using Omega3p.

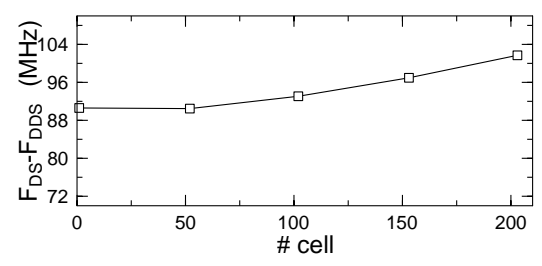

Figure 7: Frequency shift due to the damping slots.

\section{REFERENCES}

[1] JLC Design Study, KEK Report 97-001, 1997.

[2] Zeroth-order design report for the Next Linear Collider, LBNL-PUB-5424, SLAC Report 474, UCRL-ID-124161, 1996.

[3] S.G. Tantawi and et al, " A Multi-moded RF Delay Line Distribution System for the Next Linear Collider," Proceedings of EPAC98, Stockholm, June 1998.

[4] J.W. Wang and et al, "Accelerator Structure R\&D for the Linear Colliders," this conference.

[5] X. Zhan, Parallel Electromagnetic Field Solvers Using Finiteelement Method with Adaptive Refinement and Their Application to Wakefield Computation of Axisymetric Accelerator Structure, Ph.D. thesis, Stanford University, 1997.

[6] MAFIA User's Guide, CST GmbH, Darmstadt, Germany.

[7] Z. Li and et al, "OMEGA3P: A Parallel Eigensolver for the DOE Grand Challenge," this conference.

[8] R.H. Miller, Proc. LINAC96, SLAC-PUB-7288.

[9] R.M. Jones and et al, "The Transverse Long-range Wakefield in RDDS1 for the JLC/NLC X-band Linacs," this conference 